\title{
PHYSIOLOGICAL DETERMINANTS OF SHORT TRAIL RUNNING
}

\author{
Myrsini S. Kolyfa ${ }^{1}$, \\ Nikolaos D. Geladas, \\ Georgios P. Paradisis ${ }^{1}$, \\ Polyxeni Argeitaki', \\ Anastasia Tzimou², \\ Elias D. Zacharogiannis ${ }^{1}$ \\ 'School of Physical Education and Sport Science, \\ National and Kapodistrian University, \\ Athens, Greece \\ ${ }^{2}$ Laboratory of Evaluation of Human Biological Performance, \\ School of Physical Education and Sport Science, \\ Aristotle University of Thessaloniki, \\ Greece
}

\begin{abstract}
:
The recent worldwide popularity of trail running has raised the necessity of studying the physiological profile of this sport. Although trail running races are long distance endurance events, the variety of their terrain, incline and duration prevents the application of the classical predictive model of level running. Thus, the aim of the present study was to investigate the physiological and anthropometric parameters that determine short trail race performance. Twenty-five moderately trained trail runners participated in a $15 \mathrm{~km}$ trail running race, consisting of $9 \mathrm{~km}$ positive and $6 \mathrm{~km}$ negative incline. Four days after the race they followed a laboratory protocol for the measurement and estimation of anthropometric and physiological parameters (maximal oxygen uptake, velocity at maximal oxygen uptake, ventilatory threshold, velocity at ventilatory threshold, running economy, flexibility, muscle power, aerobic capacity). The results revealed high correlations between the $15 \mathrm{~km}$ race performance and velocity at maximal oxygen uptake $(\mathrm{r}=0.81)$, ventilatory threshold $(\mathrm{r}=0.88)$, muscle power of knee extensor $(\mathrm{r}=0.50-0.53)$, anaerobic capacity $(\mathrm{r}=0.65)$ and body fat percentage $(\mathrm{r}=0.7)$. Another two parameters that were highly correlated with the $15 \mathrm{~km}$ mountain trail race performance were both the positive and negative incline time $(r=0.95$ and $r=0.96$, respectively). Our conclusions confirmed previous findings that performance in trail running cannot be predicted with the same variable model as level running.
\end{abstract}

${ }^{1}$ Correspondence: email marsiak@hotmail.com 
Keywords: level running predictive model; maximal oxygen uptake; muscle power; trail running; ventilatory threshold

\section{Introduction}

Trail running (TR) is a new sport. According to the definition of the International Trail Running Association (2019), a trail race has to involve running over short to long or extreme distances on irregular terrain with large positive and negative elevation changes. The duration of the maximal effort of trail races is similar to level road and track races. However, if we consider the incline characteristics of trail courses, we can assume why TR adaptations and fatigue may differ from level courses.

The positive relationship between cardiovascular parameters such as maximal oxygen uptake $\left(\mathrm{VO}_{2}\right.$ max), ventilatory threshold (VT), running economy (RE) and endurance performance is well established (Costill et al., 1973; Daniels, 1985; Morgan et al., 1989). The majority of the studies which have investigated the prediction factors of performance in races from $5 \mathrm{~km}$ to ultramarathon support the "classical model" (Joyner, 1991) especially when they are expressed in velocity terms [velocity of maximal oxygen uptake ( $\mathrm{vVO}_{2}$ max), and velocity ventilatory threshold (vVT); Abad et al., 2016; Abe et al., 1998; Mclaughlin et al., 2010; Scott \& Houmard, 1994; Sjodin \& Jacobs, 1981; Sjodin \& Svedenhag, 1985; Stratton et al., 2009). In addition, there is evidence that body composition and especially fat-free mass is also a significant performance parameter (Bale et al., 1985; Gomez-Molina et al., 2017; Hagan et al., 1981; Knechtle et al., 2011; Oguela-Alday et al., 2018).

The difficulty in studying trail running performance is related to the diversity of terrain, incline and race distance. In particular, large uphill and downhill sections of a race provoke biomechanical changes that are related to different energy demands (Balducci et al., 2017; Vernillo et al., 2017). For this reason, the application of the "classical prediction model" is questionable (Ehrstrom et al., 2018).

Nevertheless, there are limited scientific data that present $\mathrm{VO}_{2}$ max and $\mathrm{vVO}_{2}$ max as significant prediction factors (Balducci et al., 2017) of TR performance. The role of running economy has yet to be clarified due to differences in expression terms (Balducci et al., 2017; Lazzer et al., 2014) and evaluation protocols (Balducci et al., 2017). In other prediction models, neuromuscular characteristics such as muscle stiffness, maximum isometric contraction, countermovement jump and muscle pain improve the predictive ability (Balducci et al., 2017; Ehrstrom et al., 2017). Finally, the study of body composition factors (percentage of body fat, BMI and fat-free mass) is of great interest to exercise physiology researchers and coaches because there are indices that are strongly related to trail running performance (Fornasiero et al., 2018; Hoffman, 2008)

The aim of this study was to investigate the predictive power of selected cardiovascular parameters in short trail running performance which are included in the "classical model". In addition, anaerobic power and neuromuscular variables were studied due to the intensity and race terrain characteristics. Anthropometrical and body 
composition variables were also included maintaining the hypothesis that they could improve predictive ability.

\section{Methods}

\subsection{Participants}

The study recruited twenty-five $(n=25)$ moderately trained trail runners, aged 20-50 years old, with at least 2 years of trail race experience. All participants were informed about the purpose, procedures and possible risks of the study and signed informed consent prior to the start of the experimental protocol. Moreover, all procedures were conducted in accordance with the code of Ethics of the National and Kapodistrian University of Athens, the Helsinki Declarations and the current Greek laws.

\subsection{Experimental design}

The participants ran a $15 \mathrm{~km}$ trail race of $750 \mathrm{~m}$ positive incline. The course was designed on mountain Imettus near Athens and consistent of two distinct sections, $9 \mathrm{~km}$ uphill and $6 \mathrm{~km}$ downhill while the terrain involved mainly trail paths and roads. For the race timing an electronic system with radio-frequency identification detection technology (RaceResult, Germany) was used. An intermediate checkpoint was set at $9 \mathrm{~km}$ (end of uphill section) for the separation of uphill and downhill running time estimation. Water and isotonic beverages were available at the $5^{\text {th }}$ and $9^{\text {th }} \mathrm{km}$. The participants were encouraged for maximal effort throughout the race.

\subsection{Laboratory protocol}

Four days after the $15 \mathrm{~km}$ trail race the participants visited the laboratory for the evaluation of their anthropometric characteristics (body stature, body mass, body fat percentage), cardiovascular parameters $\left(\mathrm{VO}_{2} \max , \mathrm{vVO}_{2} \max , \mathrm{VT}\right.$ and $\mathrm{vVT}$, running economy), neuromuscular parameters (maximal power of knee extensors, muscle power), anaerobic capacity and flexibility (Figure 1). They were told to refrain $48 \mathrm{~h}$ from intensified training and caffeine, as well as $4 \mathrm{~h}$ from eating.

\subsection{Anthropometry}

Body stature was measured with a Seca scale (Leicester, UK) with $1 \mathrm{~mm}$ accuracy and body mass with a Seca 710 electronic scale (Leicester, UK) with $0.01 \mathrm{~kg}$ accuracy of. Body fat percentage was measured using a Harpenden calliper (UK) at four skinfold sites, biceps, triceps, subscapular and suprailiac and the estimation was based on Durnin and Womersley equation (1974).

\subsection{Running economy (RE)}

The participants ran a 7-min warm-up on a Technogym treadmill (Technogym run race 1200 , Italy) at $0 \%$ incline followed by a 6 -min run at $10 \mathrm{~km} \cdot \mathrm{h}^{-1}$ and $6 \%$ incline for the 
determination of incline running economy. Running economy was expressed as the percentage of $\mathrm{VO}_{2}$ max used.

\section{6 $\mathrm{VO}_{2}$ max, $\mathrm{vVO}_{2}$ max, $\mathrm{VT}$, and $\mathrm{vVT}$}

The participants performed a 2-min stage incremental running test for the determination of $\mathrm{VO}_{2} \max , \mathrm{vVO}_{2} \max , \mathrm{VT}$, and $\mathrm{vVT}$. The initial speed of the treadmill (Technogym runrace 1200, Italy) corresponded to the intensity of $65 \%$ HRmax. The incline of the treadmill was kept at $0 \%$ and the velocity was increased by $1 \mathrm{~km} \cdot \mathrm{h}^{-1}$ every $2 \mathrm{~min}$. All athletes received verbal encouragement to run to voluntary exhaustion. During the last 30 seconds of each stage, expired air was collected in Douglas bags and analyzed for gas composition with the use of $\mathrm{O}_{2}$ and $\mathrm{CO}_{2}$ analyzers (Vacumed $17620 \mathrm{O}_{2} \kappa \alpha \iota 17630 \mathrm{CO}_{2}$ silver edition, USA), and for volume by using a dry gas meter (Harvard, USA) which was calibrated with a 3L syringe (Hans Rudolf 5530, Kansas City, MO). Heart rate was measured continuously with a heart rate monitor (Polar RS 100, Finland). Five minutes after the completion of the incremental test, blood lactate concentration was determined with an electroenzymatic method (Nova Biomedica, USA) on $0.7 \mu 1$ micro blood samples from the fingertip.

The following criteria were used to determine the participant's $\mathrm{VO}_{2}$ max: a) a respiratory exchange ratio $>1.05, b)$ an ending heart rate within $\pm 10 \mathrm{~b} / \mathrm{min}$ of agepredicted HRmax (220 - age), c) no further increase in $\mathrm{O}_{2}$ consumption despite the increased work rate, d) volitional exhaustion, $>18$ of Borg scale. VT was determined by a) the attenuated increase of $\mathrm{VE} / \mathrm{VO}_{2}$ without a concomitant increase of $\mathrm{VE} / \mathrm{VCO} 2$ and $\mathrm{b}$ ) an over proportional increase of VE as related to running velocity (Howley et al 1995).

\subsection{Flexibility}

The sit and reach test was used for the evaluation of flexibility. The subjects sat on the floor with their legs stretched out towards the sit and reach box (Cranlea, UK. Shoes were removed. Their feet were placed flat against the box. Both knees were locked and pressed flat to the floor (an examiner by holding them down). With their palms facing downwards and the hands-on top of each other or side by side, the participants reached forward along a measuring line as far as possible. After 3 practice trials, the participants reached out and held on that position for one to two seconds until the distance was recorded.

\subsection{Muscle power}

Muscle explosive power was measured by the vertical jumping height of a squat jump (SJ; Optojump, Microgate, Bolzano, Italy). Subjects were required to remain in a static position with a $90^{\circ}$ knee flexion angle for $2 \mathrm{~s}$, before jumping, and were instructed to execute a downward movement followed by complete extension of the legs, with the hands fixed on the hips. The obtained flight time $(t)$ was used to estimate the jump height (h; i.e., $h=\mathrm{g} \cdot \mathrm{t}^{2} / 8$ ). A total of three attempts were allowed, interspersed by $15 \mathrm{~s}$. The best attempt of the SJ was retained. 


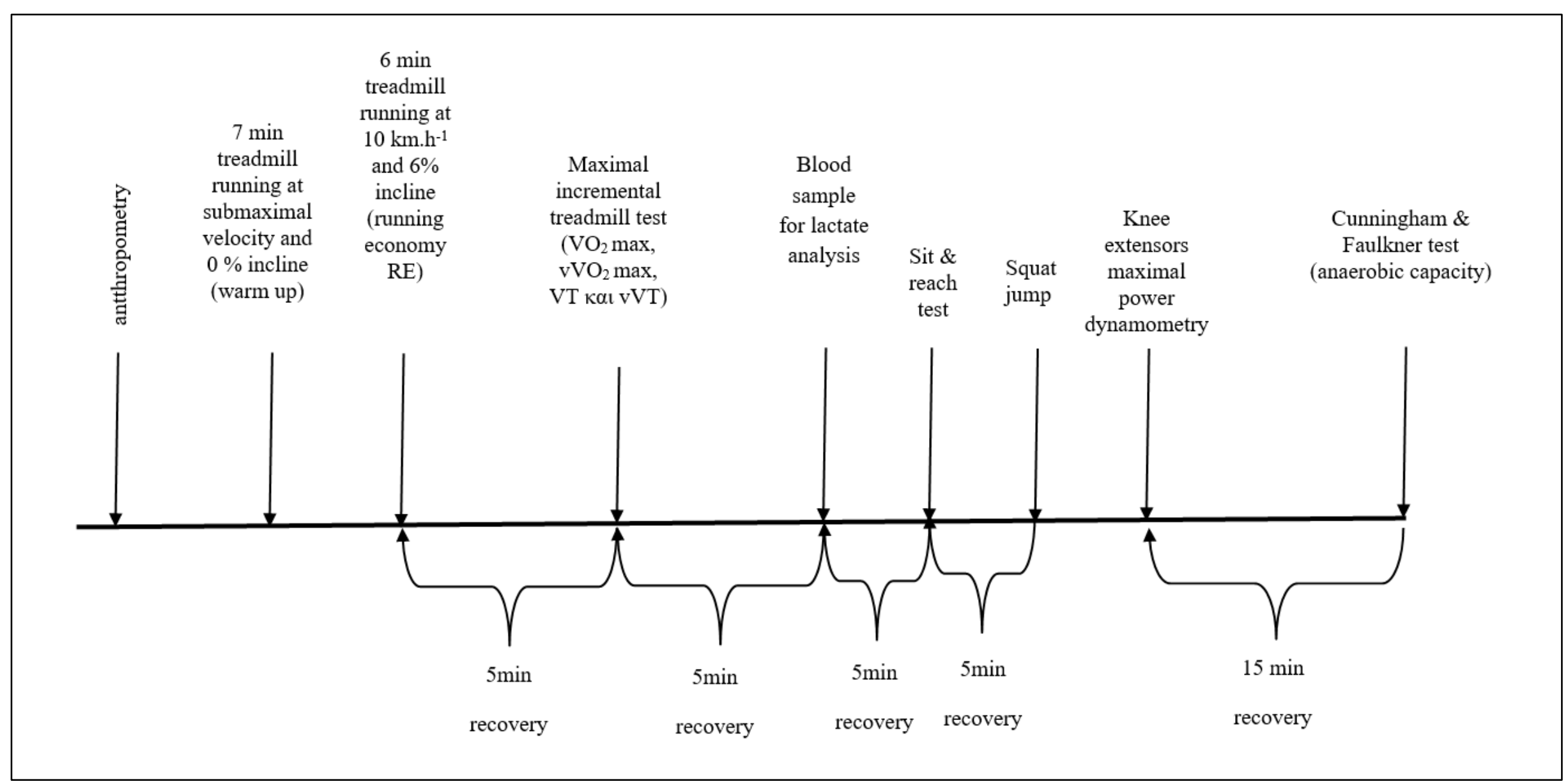

Figure 1: Laboratory protocol 


\subsection{Maximal power of knee extensors (MPKE)}

For the determination of maximal isotonic power of knee extensors and knee flexors Chronopic 3 of the Chronojump-Boscosystem (Barcelona, Spain) was used. This system records the vertical movement of a specific weight caused by the fast extension or flexion of the knee, which is then digitally analyzed through a software based on the relationship between velocity, acceleration, power and workload (Bosco et al., 1995). Participants sat in an upright position on an adjustable dynamometer chair and the shoulders, pelvis and thighs of each subject were secured by straps to minimize extraneous body movements. Before testing each participant, the knee joint was positioned at $90^{\circ}$ of flexion (full extension defined as $0^{\circ}$ ). Participants held their arms in a comfortable position during the test. Participants were given three opportunities to warm up at submaximal concentric contractions and then performed the maximal concentric contractions three times.

\subsection{Anaerobic capacity}

The anaerobic capacity of the participants was determined through the Cunningham Faulkner test (1969). After a 5-min warm-up at submaximal velocity and $0 \%$ incline they performed short familiarization runs (5-8 s) at $10-12 \mathrm{~km} \cdot \mathrm{h}^{-1}$ velocity and 6,12 and $18 \%$ incline and rested for $7 \mathrm{~min}$. Then, the treadmill was set at $13 \mathrm{~km} \cdot \mathrm{h}^{-1}$ velocity and $20 \%$ incline. The time started when the participants began to run unsupported and stopped when they grabbed the handrail. The test continued until exhaustion, meaning that they were not able to maintain the required speed. The participants received strong verbal encouragement throughout the test.

\subsection{Statistical analysis}

The results are presented as mean \pm standard deviation. The correlation analysis between variables was performed using Pearson's product-moment correlation coefficient after their normality was tested (Shapiro-Wilk test). For the interpretation of the magnitude of the correlations the following criteria were adopted: $r \leq 0.1$, trivial; $0.1<r \leq 0.3$, small; 0.3 $<r \leq 0.5$, moderate; $0.5<r \leq 0.7$, large; $0.7<r \leq 0.9$, very large; and $r>0.9$, almost perfect (Hinkle et al., 2003). Variables significantly associated with race time in the $15 \mathrm{~km}$ trail race were included in a stepwise multiple regression analysis to estimate the predictors of performance. The level of significance in all cases was set at $\alpha=0.05$.

\section{Results}

The performance details of the participants, the $15 \mathrm{~km}$ race time, $9 \mathrm{~km}$ ascent time and 6 $\mathrm{km}$ descent time are depicted in Table 1. In Table 2 the descriptive values of their anthropometric, cardiovascular and neuromuscular characteristics are shown. Table 3 contains the correlation coefficients regarding the aforementioned parameters with performance. From the anthropometric parameters only percentage of body fat was significantly correlated with the $15 \mathrm{~km}$ race time $(r=0.699, p<0.001)$. In addition, percentage of body fat was correlated significantly with all parameters except for body 
weight. The correlation coefficients of all cardiovascular parameters were also significant, especially those expressed as speed values $\left(\mathrm{vVO}_{2}\right.$ max: $r=-0.818, p<0.001$; VT: $r=0.883$, $p<0.001$ ). In addition, the correlation coefficients of $\mathrm{VO}_{2} \max , \mathrm{vVO}_{2}$ max, VT and RE with ascent time alone were higher $(r=-0.530, p<0.001, r=-0.871 ; p<0.001, r=-0.911, p<0.001$ and $r=0.744, p<0.001$, respectively). From neuromuscular parameters only MPKE was significantly correlated with the $15 \mathrm{~km}$ race time (right extensors: $r=-0.498, p<0.05$; left extensors: $r=-0.528, p<0.001)$. Flexibility was not significantly correlated with any other parameter. Squat jump was not significantly correlated with performance but showed significant correlation with maximal strength of knee extensors $(r=0.634,0.638 p<0.001)$ and flexors $(r=0.643,0.684 p<0.001)$ with percentage of body fat $(r=-0.626, p<0.001)$ and anaerobic capacity $(r=0.642, p<0.001)$. The results of the regression analysis of the parameters with the higher correlation coefficients with the $15 \mathrm{~km}$ race time are presented in Table 4 . The parameters which explained most of the performance variance were $\mathrm{vVO}_{2}$ max and VT (64\%, Figure 1 and 77\%, Figure 2, respectively). Running economy and percentage of body fat explained nearly the same percentage of variance (47 and $46 \%$ ) and anaerobic capacity (40\%). The MPKE alone explained only $21 \%$ of the total variance but in combination with $\mathrm{VVO}_{2}$ max and VT the prediction power increased to $79 \%$. The equation for the last model is: $15 \mathrm{~km}$ race time $(\mathrm{sec})=10.983,382+163,846 \mathrm{vVO}_{2} \mathrm{max}-$ 612,459 VT + 16,755 MPKE (right) + 15,475 MPKE (left) $\left(r^{2}=0.791\right.$, SEE $=401,057$ sec, $p<$ $0.001)$.

Table 1: Mountain race performance data presented as mean \pm SD

\begin{tabular}{|l|c|}
\hline Variable & Time (sec) \\
\hline Ascent & $4435.4 \pm 490.1$ \\
\hline Decent & $2394.8 \pm 425$ \\
\hline $15 \mathrm{~km}$ & $6830.3 \pm 879.1$ \\
\hline
\end{tabular}

Table 2: Anthropometric, cardiovascular and neuromuscular data presented as mean \pm SD

\begin{tabular}{|c|c|}
\hline Variable & Mean \\
\hline Mass $(\mathrm{cm})$ & $71.4 \pm 10.5$ \\
\hline Stature $(\mathrm{cm})$ & $173.8 \pm 8.6$ \\
\hline Fat $(\%)$ & $15.6 \pm 4.8$ \\
\hline $\mathrm{VO}_{2} \max \left(\mathrm{ml} \cdot \mathrm{kg}^{-1} \cdot \mathrm{min}^{-1}\right)$ & $50.4 \pm 4.7$ \\
\hline $\mathrm{vVO}_{2} \max \left(\mathrm{km} \cdot \mathrm{h}^{-1}\right)$ & $15.5 \pm 1.8$ \\
\hline $\mathrm{VT}\left(\mathrm{km} \cdot \mathrm{h}^{-1}\right)$ & $11.2 \pm 1.8$ \\
\hline $\mathrm{VO}_{2}$ max at $6 \% 10 \mathrm{~km} \cdot \mathrm{h}^{-1}(\mathrm{RE})$ & $89 \pm 6.7$ \\
\hline Anaerobic capacity (sec) & $25.6 \pm 16.5$ \\
\hline Flexibility $(\mathrm{cm})$ & $18.7 \pm 10.1$ \\
\hline Squat jump $(\mathrm{cm})$ & $29.5 \pm 4.2$ \\
\hline MP right extensors (w) & $131 \pm 36.2$ \\
\hline MP left extensors (w) & $129 \pm 37.3$ \\
\hline MP right flexors $(w)$ & $82.6 \pm 23.7$ \\
\hline MP left flexors $(w)$ & $78.2 \pm 23.7$ \\
\hline
\end{tabular}


Table 3: Correlation coefficients between anthropometric, cardiovascular, neuromuscular parameters and $15 \mathrm{~km}$ race time

\begin{tabular}{|l|c|}
\hline Variable & Pearson's $\boldsymbol{r}$ \\
\hline Mass $(\mathrm{cm})$ & -.359 \\
\hline Stature $(\mathrm{cm})$ & -.360 \\
\hline Fat $(\%)$ & $.696^{* *}$ \\
\hline $\mathrm{VO}_{2} \max \left(\mathrm{ml}^{*} \mathrm{~kg}^{-1} \cdot \mathrm{min}^{-1}\right)$ & $-.428^{*}$ \\
\hline $\mathrm{VVO}_{2} \max \left(\mathrm{km} \cdot \mathrm{h}^{-1}\right)$ & $-.818^{* *}$ \\
\hline $\mathrm{VT}\left(\mathrm{km} \cdot \mathrm{h}^{-1}\right)$ & $-.883^{* *}$ \\
\hline$\% \mathrm{VO}_{2}$ max $@ 6 \% 10 \mathrm{~km} \cdot \mathrm{h}^{-1}(\mathrm{RE})$ & $.701^{* *}$ \\
\hline Anaerobic capacity $(\mathrm{sec})$ & $-.655^{* *}$ \\
\hline Flexibility $(\mathrm{cm})$ & .295 \\
\hline Squat jump $(\mathrm{cm})$ & -.360 \\
\hline MP right extensors $(\mathrm{w})$ & $-.498^{*}$ \\
\hline MP left extensors $(\mathrm{w})$ & $-.528^{* *}$ \\
\hline MP right flexors $(\mathrm{w})$ & -.317 \\
\hline MP left flexors $(\mathrm{w})$ & -.337 \\
\hline VT: ventilatory threshold, RE: running economy, MP: muscle power & \\
\hline
\end{tabular}

Table 4: Regression analysis of the parameters with the highest correlation coefficients (VT: ventilator threshold, RE: running economy)

\begin{tabular}{|l|c|c|c|c|}
\hline Variable & Coefficient & St. Error & $\boldsymbol{P}$ & $\boldsymbol{R}^{2}$ \\
\hline $\begin{array}{l}\text { Constant } \\
\mathrm{vVO}_{2} \text { max }\end{array}$ & $\begin{array}{c}12901.738 \\
-392.820\end{array}$ & 517.191 & 0.000 & 0.635 \\
\hline $\begin{array}{l}\text { Constant } \\
\mathrm{VT}\end{array}$ & $\begin{array}{c}11697.668 \\
-433.038\end{array}$ & 422.159 & 0.000 & 0.769 \\
\hline $\begin{array}{l}\text { Constant } \\
\% \mathrm{VO}_{2} \text { max } \\
@ \begin{array}{l}\text { @ } \% 10 \mathrm{~km} \cdot \mathrm{h}^{-1} \\
\text { (RE) }\end{array}\end{array}$ & $\begin{array}{c}-1292.173 \\
91.252\end{array}$ & 640.714 & 0.000 & 0.468 \\
\hline $\begin{array}{l}\text { Constant } \\
\text { Anaerobic capacity }\end{array}$ & $\begin{array}{l}7719.575 \\
-34.790\end{array}$ & 678.542 & 0.000 & 0.404 \\
\hline $\begin{array}{l}\text { Constant } \\
\% \text { Fat }\end{array}$ & $\begin{array}{c}4846.303 \\
127.507\end{array}$ & 645.128 & 0.000 & 0.461 \\
\hline
\end{tabular}




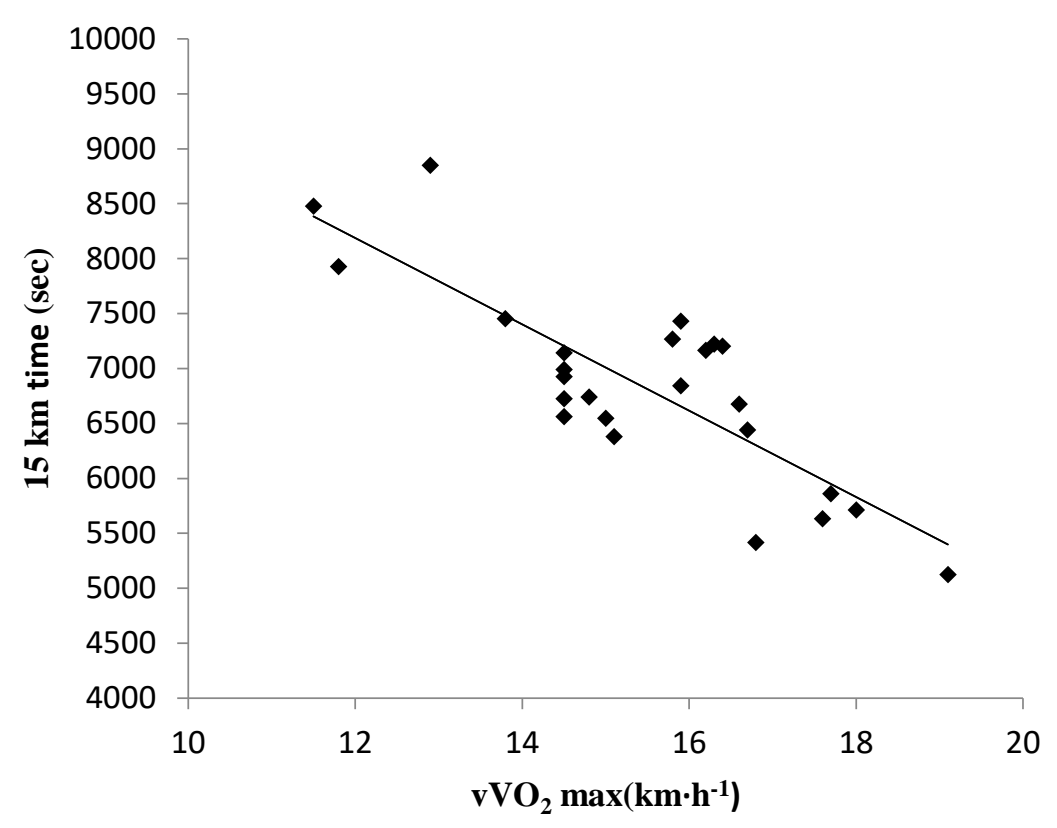

Figure 1: Scatter plot of the correlation between $\mathrm{vVO}_{2}$ max and performance time of the $15 \mathrm{~km}$

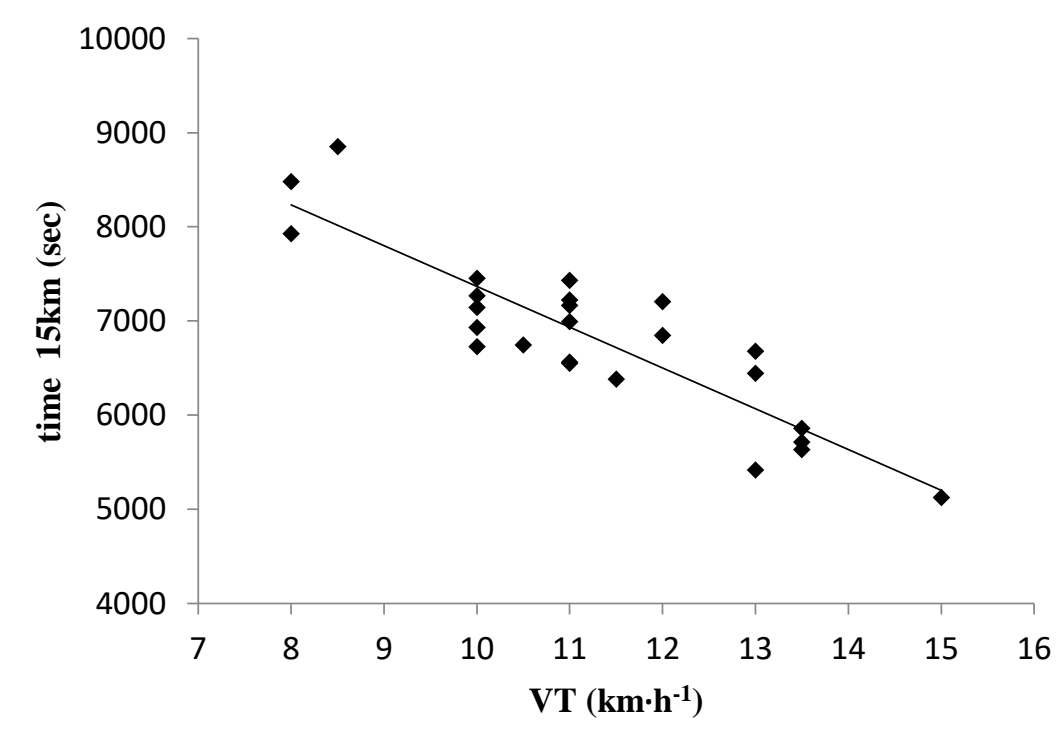

Figure 2: Scatter plot of the correlation between VT and performance time of the $15 \mathrm{~km}$

\section{Discussion}

The main finding of the study was that short trail running performance can be accurately predicted by a combination of the physiological parameters of the "classical model" and neuromuscular characteristics that allow athletes to sustain pacing through the demanding trail terrain. The fact that $\mathrm{VVO}_{2}$ max and VT were highly correlated with the $15-\mathrm{km}$ race time ( $r=0.818$ and 0.883 , respectively) is something that has been strongly confirmed in level races of the same duration (Abe et al., 1998; Cunningham, 1990; Mclaughlin et al., 2010; Stratton et al., 2009; Yoshida et al., 1993). Among the few scientific 
data regarding trail races, Balducci et al. (2017) reported the same high coefficient $(r=-$ 0.74) between maximal aerobic speed and $75 \mathrm{~km}$ race time and Fornasiero et al. (2018) between power max (the result of speed, angle of inclination and gravital acceleration of the treadmill) and $65 \mathrm{~km}$ race time $(r=-0.73)$. In the study of Alvero Cruz et al. (2019) $\mathrm{vVO}_{2}$ max predicted $60 \%$ of the $27-\mathrm{km}$ race time $(r=-0.776)$ closely to our results $\left(R^{2}=\right.$ $64 \%)$. Although there aren't any data regarding the association between VT and trail performance, Sheer et al. (2019) tried to investigate the predictive power of lactate thresholds in short trail races. In particular, they found that LT4 (lactate threshold at $4 \mathrm{mmol} / \mathrm{l})$ could strongly predict $\left(R^{2}=0.753\right)$ a $31.1-\mathrm{km}$ race time. The importance of speed in terms of physiological variables is undisputed because they reflect the transformation of metabolic power to mechanical power (Ettema \& Loràs, 2009), representing one of the main determinants of endurance performance (Joyner \& Coyle, 2008).

Maximal strength of knee extensors together with $\mathrm{vVO}_{2}$ max and VT optimized the predictive power of our model to 79\%. Similarly, Balducci et al. (2019) combined maximal aerobic speed and knee extensors force but reported a higher coefficient of determination, $R^{2}=0.98$. Nevertheless, the race which had been studied was longer (75 $\mathrm{km} 3700$ positive and negative incline) and the role of neuromuscular fatigue is supposed to be higher (Giandolini et al., 2016). Ehrstrom et al. (2018) estimated a muscle fatigue index determined by averaging maximal concentric torque values and found that together with $\mathrm{VO}_{2}$ max and running economy at $10 \%$ incline could predict the performance of a $27-\mathrm{km}$ race to $98 \%$. The importance of neuromuscular characteristics in trail racing is warranted because there are findings that uneven terrain of steep up hills and down hills increases the recruitment of vastus lateralis (Vernillo et al., 2017) and provokes fatigue similar to resistance and eccentric training (Balsalobre-Fernandez, Santos-Concejero \& Grivas, 2015).

Running economy did not significantly improve the predictive power of our model although the correlation coefficient was high $(r=0.7)$. The truth is that there are many differences between studies in expression terms and units regarding running economy that makes the comparison between the results difficult. In the present study, running economy was expressed as the $\%$ of $\mathrm{VO}_{2}$ max of the participants when running at $10 \mathrm{~km} \cdot \mathrm{h}^{-1}$ speed and 6\% positive incline. Lazzer et al. (2014) used the metabolic cost of transport $\left(\mathrm{VO}_{2} \cdot \mathrm{kg}^{-1} \cdot \mathrm{m}^{-1}\right)$ which was measured at $10 \mathrm{~km} \cdot \mathrm{h}^{-1}$ and $0 \%$ incline and found a higher coefficient $(r=0.64)$. Erhstrom et al. (2018) found that only when running economy was estimated through an incline protocol and expressed in units of energy $\left(\mathrm{J} \cdot \mathrm{kg}^{\cdot-1} \cdot \mathrm{m}^{-1}\right.$ at $9 \mathrm{~km} \cdot \mathrm{h}^{-1}$ and $10 \%$ positive incline) increased the predictive power of their model. Furthermore, in the aforementioned studies the participants ran longer races (3 days race and $27-\mathrm{km}$ race, respectively) and it is possible that the role of running economy depends on the duration of the race.

Another similar finding with the existing scientific data is that $\%$ fat was significantly correlated with the $15-\mathrm{km}$ trail race time. The importance of low values of $\%$ fat in endurance running performance is well established in level races ranging from half marathon to marathon (Bale et al., 1985; Dotan et al., 1983; Gomez-Molina et al., 2017; 
Hagan et al., 1981; Knechtle et al., 2011; Ogueta-Alday et al., 2018). There is only one study that reported a high correlation $(r=0.711)$ of $\%$ of body fat with a $27-\mathrm{km}$ tail race (Alvero Cruz et al., 2019). Furthermore, when \% of body fat was combined with $\mathrm{VO}_{2}$ max the predictive power of the model was increased from 59 to $83 \%$. We suppose that these findings are related to the increased positive external mechanical work during up hills associated with the replacement of centre of mass and the advantage that low adiposity athletes have in the propulsion of the body (Vernillo et al., 2017).

Regarding the role of anaerobic capacity, the present study has shown that it is correlated significantly with the $15-\mathrm{km}$ trail race performance $(r=-0.655)$ without increasing the predictive power of the model. The importance of anaerobic capacity in trail races is maybe related to the intensity that can be sustained by the athletes which depends also on their competitive level (Fornasiero et al., 2018). In particular, the shorter the race the more time is spent above the anaerobic threshold (Fornasiero et al., 2017; Ramos-Campo et al., 2016). However, in the study of Rodriguez-Marroyo et al. (2018) it is reported that regardless the duration of the trail race (from $10 \mathrm{~km}$ to ultramarathon) the time spent above the anaerobic threshold is similar between the races (50 min) which means that there is always an anaerobic component that has to be considered when training plan is administrated. Furthermore, the participants in our study were moderately trained and not elite athletes who are supposed to run longer in higher ventilatory demands (Esteve-Lanao, 2005).

Contrary to other studies, we did not find a high correlation coefficient between $\mathrm{VO}_{2}$ max and $15-\mathrm{km}$ trail race time $(r=-0.428, p<0.05)$. Lazzer et al. (2014) reported a high correlation $(r=0.79)$ between $\mathrm{VO}_{2}$ max and 3-day $(25,55,13 \mathrm{~km})$ trail race and Ehrstrom et al. (2018) between $\mathrm{VO}_{2}$ max and a $27-\mathrm{km}$ trail race $(r=-0.76)$. A similar coefficient $(r=-$ 0.757) was demonstrated by Alvero Cruz et al. (2019) who also studied a 27-km trail race. In the study of Fornasiero et al. (2018) the correlation coefficient between $\mathrm{VO}_{2}$ max and $65-\mathrm{km}$ trail race performance was -0.66 and it is claimed that the differences between studies are related to the heterogeneity of the maximal values of the participants (Joyner \& Coyle, 2008). However, according to Sheer et al. (2019) $\mathrm{VO}_{2}$ max continues to be an important predictive factor of performance even in homogenous groups and ultramarathons because it is a marker of how efficiently the metabolic substrates can be used in submaximal intensities (Millet et al., 2011). The differences in the present study could be attributed to the level of the participants or the short duration of the race.

In conclusion, the present study confirms the importance of cardiovascular parameters of the classical model, especially when they are expressed in speed units $\left(\mathrm{vVO}_{2} \mathrm{max}, \mathrm{VT}\right)$ for the prediction of short trail-running performance. The optimization of the prediction cannot be achieved unless the neuromuscular characteristics of the maximal power of knee extensors are included. The latter is warranted if we consider the biomechanical changes that are induced by the uneven trail terrain of up-hills and downhills. 


\title{
Conflict of Interest Statement
}

The authors declare no conflicts of interests.

\begin{abstract}
About the Authors
Myrsini S. Kolyfa is a Physical Education teacher in Secondary Education in Greece who completed her PhD studies at the School of Physical Education and Sport Science of the National and Kapodistrian University of Athens. Her research about trail running was a result of her academic interests in exercise physiology and her personal athletic experience as a trail runner in marathon and ultramarathon trail races.

Nikolaos D. Geladas is a Professor at the School of Physical Education and Sport Science of the National and Kapodistrian University of Athens. His research interests are in the areas of human performance in adverse environmental conditions, and in factors of fatigue during prolonged exercise.
\end{abstract}

Georgios P. Paradisis is an Associate Professor at the School of Physical Education and Sport Science of the National and Kapodistrian University of Athens. His research interests are in the areas of exercise physiology and biomechanics, as well as in physical activity and health.

Polyxeni Argeitaki is an Assistant Professor at the School of Physical Education and Sport Science of the National and Kapodistrian University of Athens. Her research interests are in the areas of track and field in infancy and childhood, also, in factors that contribute to the improvement of the performance of track and field athletes, and in studying the long-term health status of long-distance runners.

Anastasia Tzimou is a Dr. in Exercise Biochemistry and is a member of the Laboratory of Evaluation of Human Biological Performance at the School of Physical Education and Sport Science of the Aristotle University of Thessaloniki. Her research interests are in the areas of exercise biochemistry and physiology, as well as in physical activity and health. Elias Zacharogiannis is an Associate Professor at the School of Physical Education and Sport Science of the National and Kapodistrian University of Athens. His research interests are in the areas of muscle glycogen metabolism at submaximal running speed, and in the effects of high-intensity interval training on endurance performance.

\section{References}

Abad C, Barros RV, Bertuzzi R, Gagliardi JF, Lima-Silva AE, Lambert MI, Pires FO. 2016. $10 \mathrm{~km}$ running performance predicted by a multiple linear regression model with allometrically adjusted variables. Journal of Human Kinetics, 2(51):193-200. doi: 10.1515/ hukin-2015-0182

Abe D, Yanagawa K, Yamanobe K, Tamura K. 1998. Assessment of middle-distance running performance in sub-elite young runners using energy cost of running. European Journal of Applied Physiology and Occupational Physiology, 77(4): 320-325. doi: $10.1007 / \mathrm{s} 004210050340$ 
Alvero-Cruz JR, Parent Mathias V, Garcia Romero J, Carrillo de Albornoz-Gil M, BenítezPorres J, Ordoñez FJ, Rosemann T, Nikolaidis PT, Knechtle B. 2019. Prediction of Performance in a Short Trail Running Race: The Role of Body Composition. Frontiers in Physiology, 16(10):1306. doi: 10.3389/fphys.2019.01306

Balducci P, Clemencon M, Trama R, Blache Y, Hautier C. 2017. Performance factors in a mountain ultramarathon. International Journal of Sports Medicine, 38(11): 819-826. doi:10.1055/s-0043-112342

Balducci P, Saboul D, Trama R. 2019. Monitoring heart rates to evaluate pacing on a 75km MUM. Journal of Sports Medicine and Physical Fitness, 59(7):1133-1137. doi: 10.23736/S0022-4707.18.08861-8

Bale P, Rowell S, Colley E. 1985. Anthropometric and training characteristics of female marathon runners as determinants of distance running performance. Journal of Sports Sciences, 3(2): 115-126. doi: 10.1080/02640418508729741

Balsalobre-Fernandez C, Santos-Concejero J, Grivas GV. 2015. The effects of strength training on running economy in highly trained runners: a systematic review with meta-analysis of controlled trials. Journal of strength and Conditioning Research, doi:10.1519/JSC.0000000000001316.

Bosco C, Belli A, Astrua M, Tihanyi J, Pozzo R, Kellis S, Tsarpela O, Foti C, Manno R, Tranquilli C. 1995. A dynamometer for evaluation of dynamic muscle work. European Journal of Applied Physiology and Occupational Physiology, 70(5): 379386.doi:10.1007/BF00618487

Costill DL, Thomason H, Roberts E. 1973. Fractional utilization of the aerobic capacity during distance running. Medicine and Science in Sports and Exercise, 5(4):248-252. PMID:4774203

Cunningham DA, Faulkner JA. 1969. The effect of training on aerobic and anaerobic metabolism during a short exhaustive run. Medicine and Science in Sports, 1(2): 6569. doi: 10.1249/00005768-196906000-00002

Cunningham LN. 1990. Relationship of running economy, ventilator threshold, and maximal oxygen consumption to running performance in high school females.

Research Quarterly for Exercise and Sport, 61(4): 369-374. doi: 10.1080/02701367.1990.10607501

Daniels JT. 1985. A physiologist's view of running economy. Medicine and Science in Sports and Exercise, 17(3): 332-338. PMID: 3894870

Dotan R, Rotstein A, Dlin R, Inbar O, Kofman H, Kaplansky Y. 1983. Relationships of marathon running to physiological, anthropometric and training indices. European journal of Applied Physiology and Occupational Physiology, 51(2): 281-293. doi: 10.1007/BF00455191

Ehrstrom S, Tartaruga MP, Easthope CS, Brisswalter J, Morin JB, Vercruyssen F. 2018. Short trail running race: Beyond the classic model for endurance running performance. Medicine and Science in Sports and Exercise, 50(3): 580-588. doi: 10.1249/MSS.0000000000001467 
Esteve-Lanao J, San Juan AF, Earnest CP, Foster C, Lucia A. 2005. How do endurance runners actually train? Relationship with competition performance. Medicine and Science in Sports and Exercise, 37(3): 496-504. doi: 10.1249/01.mss.0000155393.78744.86

Ettema G, Loras HW. 2009. Efficiency in cycling: a review. European Journal of Applied Physiology, 106(1): 1-14. doi: 10.1007/s00421-009-1008-7

Fornasiero A, Savoldelli A, Fruet D, Boccia C, Pellegrini B, Schena F. 2018. Physiological intensity profile, exercise load and performance predictors of a $65-\mathrm{km}$ mountain ultra-marathon. Journal of Sports Sciences, 36(11): 1287-1295. doi: 10.1080/02640414.2017.1374707

Giandolini M, Vernillo G, Samozino P, Horvais N, Edwards WB, Morin JB, Millet GY. 2016. Fatigue associated with prolonged graded running. European Journal of Applied Physiology. 116(10):1859-1873. doi: 10.1007/s00421-016-3437-4

Gomez-Molina J, Ogueta-Alday A, Camara J, Stickley C, Rodriguez-Marroyo JA, GarciaLopez J. 2017. Predictive Variables of Half-Marathon Performance for Male Runners. Journal of Sports Sciences and Medicine, 16(2):187-194. PMCID: PMC5465980

Hagan RD, Smith MG, Gettman LR. 1981. Marathon performance in relation to maximal aerobic power and training indices. Medicine and Science in Sports and Exercise, 13(3):185-189. PMID: 7253871

Hoffman MD. 2008. Anthropometric characteristics of ultramarathoners. International Journal of Sports Medicine, 29(10):808-811. doi: 10.1055/s-2008-1038434

Howley ET, Bassett DRJ, Welch HG. 1995. Criteria for maximal oxygen uptake: review and commentary. Medicine and Science in Sports and Exercise, 27(9):1292-1301. PMID: 8531628

Joyner MJ. 1991. Modeling: optimal marathon performance on the basis of physiological factors. Journal of Applied Physiology, 70(2):683-687. doi: 10.1152/jappl.1991.70.2.683

Joyner MJ, Coyle EF. 2008. Endurance exercise performance: the physiology of champions. The Journal of Physiology, 586(1):35-44. doi: 10.1113/jphysiol.2007.143834

Knechtle B, Knechtle P, Barandun U, Rosemann T, Lepers R. 2011. Predictor variables for half marathon race time in recreational female runners. Clinics, 66(2): 287-291. doi: 10.1590/s1807-59322011000200018

Knechtle B, Knechtle P, Rosemann T, Lepers R. 2011. Personal best marathon time and longest training run, not anthropometry, predict performance in recreational 24hour ultrarunners. Journal of Strength and Conditioning Research, 25(8): 2212-2218. doi: 10.1519/JSC.0b013e3181f6b0c7

Lazzer S, Taboga P, Salvadego D, Rejc E, Simunic B, Narici MV, Bulgione A, Giovanelli N, Antonutto G, Grassi B, Pisot R, Di Prampero PE. 2014. Factors affecting metabolic cost of transport during a multi-stage running race. The Journal of Experimental Biology, 217(Pt 5):787-795. doi: 10.1242/jeb.091645 
McLaughlin JE, Howley TE, Bassett DR Jr, Thompson DL, Fitzhugh EC. 2010. Test of the classic model for predicting endurance running performance. Medicine and Science in Sports and Exercise, 42(5): 991-997. doi: 10.1249/MSS.0b013e3181c0669d

Millet GY, Tomazin K, Verges S, Vincent C, Bonnefoy R, Boisson RC, Gergele L, Feasson L, Martin V. 2011. Neuromuscular consequences of an extreme mountain ultramarathon. PLoS One, 6(2): e17059. doi: 10.1371/journal.pone.0017059

Morgan DW, Baldini FD, Martin PE, Kohrt WM. 1989. Ten-kilometer performance and predicted velocity at VO2max among well-trained male runners. Medicine and Science in Sports and Exercise, 21(1): 78-83. doi: 10.1249/00005768-198902000-00014

Ogueta-Alday A, Morante JC, Gomez-Molina J, Garcia-Lopez J. 2018. Similarities and differences among half-marathon runners according to their performance level. PLoS One, 13(1): 1-11. doi: 10.1371/journal.pone.0191688

Ramos-Campo DJ, Ávila-Gandía V, Alacid F, Soto-Méndez F, Alcaraz PE, López-Román FJ, Rubio-Arias JÁ. 2016. Muscle damage, physiological changes, and energy balance in ultra-endurance mountain-event athletes. Applied Physiology of Nutrition and Metabolism, 41(8): 872-878. doi: 10.1139/apnm-2016-0093

Rodríguez-Marroyo JA, González-Lázaro J, Arribas -Cubero HF, Villa JG. 2018. Physiological demands of mountain running races. Kinesiology, 50(1). doi: 10.1016/j.injury.2020.10.045

Scheer V, Vieluf S, Janssen TI, Heitkamp H. 2019. Predicting Competition Performance in Short Trail Running Races with Lactate Thresholds. Journal of Human Kinetics, 69: 159-167. doi: 10.2478/hukin-2019-0092

Scott BK, Houmard JA. 1994. Peak running velocity is highly related to distance running performance. International Journal of Sports Medicine, 15(8): 504-507. doi: 10.1055/s2007-1021095

Sjodin B, Jacobs I. 1981. Onset of blood lactate accumulation and Marathon running performance. International Journal of Sports Medicine, 2(1): 23-26. doi: 10.1055/s2008-1034579

Sjodin B, Svedenhag J. 1992. Oxygen uptake during running as related to body mass in circumpubertal boys: a longitudinal study. European Journal of Applied Physiology, 65(2):150-157. doi: 10.1007/BF00705073

Stratton E, O'Brien BJ, Harvey J, McNicol AJ, Janissen D, Paton C, Knez W. 2009. Treadmill velocity best predicts 5000-m run performance. International Journal of Sports Medicine, 30(1): 40-45. doi: 10.1055/s-2008-1038761

Vernillo G, Giandolini M, Edwards WB, Morin JB, Samozino P, Horvais N, Millet GY. 2017. Biomechanics and physiology of uphill and downhill running. Sports Medicine, 47(4): 615-629. doi: 10.1007/s40279-016-0605-y

Yoshida T, Udo M, Iwai K, Yamaguchi T. 1993. Physiological characteristics related to endurance running performance in female distance runners. Journal of Sports Sciences, 11(1): 57-62. doi: 10.1080/02640419308729964 

copy, distribute, transmit or adapt the article content, providing a proper, prominent and unambiguous attribution to the authors in a manner that makes clear that the materials are being reused under permission of a Creative Commons License. Views, opinions and conclusions expressed in this research article are views, opinions and conclusions of the author(s). Open Access Publishing Group and European Journal of Physical Education and Sport Science shall not be responsible or answerable for any loss, damage or liability caused in relation to/arising out of conflict of interests, copyright violations and inappropriate or inaccurate use of any kind content related or integrated on the research work. All the published works are meeting the Open Access Publishing requirements and can be freely accessed, shared, modified, distributed and used in educational, commercial and non-commercial purposes under a Creative Commons attribution 4.0 International License (CC BY 4.0). 